\title{
Netrin-1-Dependent Spinal Interneuron Subtypes Are Required for the Formation of Left-Right Alternating Locomotor Circuitry
}

\author{
Nadine Rabe, Henrik Gezelius, ${ }^{\star}$ Anna Vallstedt, ${ }^{\star}$ Fatima Memic, and Klas Kullander \\ Department of Neuroscience, Uppsala University, 75124 Uppsala, Sweden
}

\begin{abstract}
Neuronal circuits in the spinal cord that produce the rhythmic and coordinated activities necessary for limb movements are referred to as locomotor central pattern generators (CPGs). The identities and preceding development of neurons essential for coordination between left and right limbs are not yet known. We show that the ventral floor plate chemoattractant Netrin-1 preferentially guides dorsally originating subtypes of commissural interneurons, the majority of which are inhibitory. In contrast, the excitatory and ventralmost V3 subtype of interneurons have a normal number of commissural fibers in Netrin-1 mutant mice, thus being entirely independent of Netrin-1-mediated attraction. This selective loss of commissural fibers in Netrin-1 mutant mice resulted in an abnormal circuitry manifested by a complete switch from alternating to synchronous fictive locomotor activity suggesting that the most ventral-originating excitatory commissural interneurons are an important component of a left-right synchrony circuit in the locomotor CPG. Thus, during development, Netrin-1 plays a critical role for the establishment of a functional balanced CPG.
\end{abstract}

\section{Introduction}

The spinal cord central pattern generator $(\mathrm{CPG})$ that controls locomotion is a promising system to dissect neuronal circuit function. Previous studies have shown that the axon guidance molecules EphA4 and EphrinB3 cooperate to prevent ipsilateral interneurons from crossing the midline in the spinal cord and if either molecule is deleted in mice, this will result in a synchronous gait (Kullander et al., 2003). Netrin-1, another axon guidance molecule, acts as a diffusible floor plate chemotrophic cue for commissural axons (Kennedy et al., 1994). Binding of Netrin-1 to its receptor DCC mediates an attractive effect to growing axons, and in Netrin-1-deficient mice, the majority of dorsal commissural axons no longer find and cross the midline (Serafini et al., 1996). However, the consequence of Netrin-1 deletion for CPG functionality is not yet clear.

Commissural interneurons (CINs) in the spinal cord are considered to participate in bilateral coordination during locomotion (for review, see (Grillner, 2003). A working model for left-right coordination has been suggested to consist of one circuit responsible for left-right alternation and, in addition, one circuit responsible for left-right synchrony (Quinlan and

\footnotetext{
Received 0ct. 13, 2009; accepted 0ct. 30, 2009.

*H.G. and A.V. contributed equally to this work.

This work was supported by grants from the Swedish Medical Research Council (2004-5567, 2007-3630/4479, K2005-33X-15327), the International Institute for Research on Paraplegia, the Swedish Brain Foundation, the foundations of Knut and Alice Wallenberg, Åke Wiberg, Magnus Bergwall, Åhlén, and Hedlund, and Uppsala University. K.K. is a Royal Swedish Academy of Sciences Research Fellow supported by a grant from the Knut and Alice Wallenberg Foundation. We thank M. Tessier-Lavigne for Netrin-1 mice, and A. Enjin, M. Goulding, and A. Klar for helpful comments.

Correspondence should be addressed to Klas Kullander, Department of Neuroscience, Uppsala University, Box 593, 75124 Uppsala, Sweden. E-mail: klas.kullander@neuro.uu.se.

DOI:10.1523/JNEUROSCI.5096-09.2009

Copyright $\odot 2009$ Society for Neuroscience $\quad$ 0270-6474/09/2915642-08\$15.00/0
}

Kiehn, 2007). Experiments using neonatal spinal cord preparations have suggested that the components of the locomotor CPG reside in the ventral spinal cord (Kjaerulff and Kiehn, 1996). Nevertheless, during development, ventral migration of dorsally born neurons has been reported (Bermingham et al., 2001; Pierani et al., 2001; Gross et al., 2002; Lanuza et al., 2004), implying that neurons originating from both the ventral and dorsal spinal cord may contribute to the CPG.

Subpopulations of spinal cord interneurons can be identified during development by their expression of specific homeodomain transcription factors (Jessell, 2000; Goulding et al., 2002). Dorsal progenitor cells give rise to six early classes of neurons, dI1 to dI6, and ventral progenitors give rise to motor neurons and four classes of interneurons, V0 to V3. Several of the ventraloriginating populations have recently been shown to be involved in specific aspects of locomotion (Gosgnach et al., 2006; Crone et al., 2008; Zhang et al., 2008). With regard to left-right coordination, the rhythms of fictive locomotion were irregular with episodes of synchrony and alternation (Lanuza et al., 2004) or drifting in and out of strict alternation (Crone et al., 2008), perhaps best described as uncoordinated phenotypes. Consequently, it seems likely that multiple neuronal subtypes originating from several progenitor domains are involved in the different aspects of left-right coordination.

Given its role in guiding neurons toward the midline, Netrin- 1 is an attractive candidate for providing guidance cues to CINs originating from multiple developmental domains. We show that in mice lacking Netrin-1 only a few CINs predominantly originating from the most ventral progenitor domains find and cross the midline and that these CINs are mostly excitatory. Functionally, Netrin-1 deletion leads to a strict synchronous left-right coordination during fictive locomotion. Our data thus 
suggest a role for ventral-originating excitatory CINs as part of the circuit controlling synchronous activities over the midline.

\section{Materials and Methods}

Animals and genotyping. Netrin-1 (Ntn1 Gt(pGT1.8TM)629Wcs) mice were maintained on C57BL/6J background (Serafini et al., 1996). Mutant mice were received from heterozygous crosses and were identified by their abnormal phenotypes (body posture, abnormal limb flexion, and synchronous forelimb movements) or for embryos, by in situ hybridization. All experiments involving animals were approved by the appropriate local Swedish ethical committee (C147/7).

Tracing of commissural interneurons. Fluorescent dextran-amines 3000 MW rhodamine-dextran-amine (RDA) and 3000 MW fluoresceindextran-amine (FDA) (Invitrogen) were used for retrograde tracing of CINs as described previously (Eide and Glover, 1995). Postnatal day 0 (P0)-P3 mice spinal cords were prepared as described earlier (Wegmeyer et al., 2007). Tracings on embryonic day 12.5 (E12.5) embryos were performed essentially the same way, but the spinal cord remained within the vertebral column during tracer application and incubation (see also Nissen et al., 2005). Preparations were incubated for $12-16 \mathrm{~h}$ and then fixed in $4 \%$ paraformaldehyde (PFA) in $0.1 \mathrm{M} \mathrm{PBS,} \mathrm{pH} 7.4$ and stored dark at $4^{\circ} \mathrm{C}$ for 1 week. Spinal cords were cut into 60 - $\mu \mathrm{m}$-thick transverse sections on a vibratome (Leica) and stored in the dark at $-20^{\circ} \mathrm{C}$ until analysis. Samples for in situ hybridization and immunohistochemistry studies were fixed for $2 \mathrm{~h}$ and then transferred to $30 \%$ sucrose in PBS at $4^{\circ} \mathrm{C}$ overnight. Embryonic tracings and samples were embedded in OCT and $12 \mu \mathrm{m}$ sections were cut using a cryostat (CM1800, Leica), collected onto Superfrost slides (Menzel-Gläser) and stored in the dark at $-80^{\circ} \mathrm{C}$ until usage for additional staining. Only preparations where the spinal cord midline was intact, as judged by inspection of sections from the tracer application sites, were used for analysis.

Spinal cord electrophysiology. Electrophysiological experiments were performed on isolated spinal cords according to previously described procedures (Wegmeyer et al., 2007) except for the specifications stated below. All mice were killed at P0. A combination of $N$-methyl-Daspartate (NMDA, 4-10 $\mu \mathrm{M}$ ), serotonin (5-HT, 3-10 $\mu \mathrm{M}$ ), and dopamine $(50 \mu \mathrm{M}$, not always added) were added to the perfusing artificial CSF (aCSF) to induce stable locomotor-like output. When the glycine reuptake inhibitor sarcosine $(100 \mu \mathrm{M})$ was used, it was added to the aCSF together with neurotransmitters. The grand means for all animals before and after treatment were calculated. In these comparisons, only the animals receiving the same treatment were included.

In situ hybridization. In situ hybridization on cryosections was performed essentially as described previously (Schaeren-Wiemers and Gerfin-Moser, 1993). The Netrin probe covers nucleotides 3183-5750 (GeneID: 22348/NM_009508.2), the VIAAT probe covers nucleotides 588-2072 (GeneID: 18208/NM_008744.2) and the Vglut2 probe was used as described earlier (Wallén-Mackenzie et al., 2006).

Immunohistochemistry. Antibodies on E12.5 embryo traced spinal cords were used in 5\% goat serum, $0.3 \%$ BSA in PBS in the following dilutions: mNkx2.2 1:100 (Developmental Studies Hybridoma Bank, University of Iowa, Iowa City, IA), mEvx1 1:50 (Developmental Studies Hybridoma Bank), gpLbx1 1:10,000 (kind gift from C. Birchmeier, MDC, Berlin, Germany), rPax2 1:1000 (Covance) mBrn3a 1:500 (Millipore Bioscience Research Reagents) 1:100 mIsl1 (), 1:500 mLhx1/5 (Developmental Studies Hybridoma Bank) and 1:8000 Lhx2/9 (kind gift from T. Jessell, Columbia University, New York, NY).

Imaging and picture processing. Fluorescent and bright field images were viewed in an Olympus BX61WI microscope. For quantitative analyses of traced spinal cords, the application sites were identified and photographs were taken on sections between the two application sites. Higher-magnification pictures were taken using the OptiGrid Grid Scan Confocal Unit (Qioptiq) and Volocity software (Improvision). In situ hybridization pictures were taken in bright field and pseudo-colored for colabeling analyses. Captured images were auto-leveled using Adobe Photoshop software.

\section{Results}

Commissural interneuron numbers are greatly reduced in Netrin-1 mutant mice

It has been shown earlier that fewer prospective commissural axons reach the floor plate in Netrin-1 mutant mice (here referred to as $N t n 1^{G t / G t}$ ) E11.5 embryonic spinal cords (Serafini et al., 1996). To determine the extent to which CINs are affected in $N t n 1^{G t / G t}$ mice, we analyzed spinal cords at age E12.5 using a modified embryonic tracing method (Fig. 1) (Eide and Glover, 1995). In controls, the majority of the CINs were found in a tight cluster in the ventromedial region and in two more clusters of traced neurons more dorsolateral of this group, which is comparable to results obtained previously (Fig. 1E) (Nissen et al., 2005). The traceable CINs in the spinal cords of $N t n 1^{G t / G t}$ embryos were significantly fewer, affecting the ventromedial and dorsolateral populations. In control embryo spinal cords, we found an average of 42 traced neurons/section (10,616 total neurons, $257 \mathrm{sec}-$ tions, 15 embryos) whereas in $N t n 1^{G t / G t}$ embryos we found 11 traced neurons/section $(1710,154,5)$, which is a reduction to $28 \%$ of the control levels (Fig. 1 E, F; $p<0.0001$, Mann-Whitney test). The traced neurons remaining in $N t n 1^{G t / G t}$ embryos had a similar morphology and orientation toward the midline compared with the traced neurons in control embryos.

The dynamic maturation and differentiation of spinal cord neurons continues after E12.5, and it was uncertain to what extent the reduced number of commissural neurons in Netrin-1 mutant mice detected at E12.5 is compensated for during development. We therefore next investigated short- and long-range projecting CINs by intra- and intersegmental tracer application in $\mathrm{P} 0$ mice (Fig. $2 \mathrm{~A}$ ). At later neonatal stages, rodent CINs can be divided into four subpopulations based on their pattern of projections (ascending, descending, bifurcating or local axonal fibers) (Stokke et al., 2002). Locally short projecting CINs, together with long-range projecting CINs, were detected by examination of intrasegmental tracings, and were found severely reduced in $N t n 1^{G t / G t}$ mice compared with controls (Fig. $2 B, C$ ). To study the P0 long-range projecting CINs in further detail, we examined three different subpopulations; the intersegmental ascending (a), descending (d), and bifurcating (ad) CINs (Fig. 2A). In control neonatal mice, our tracing experiments showed that aCINs and dCINs comprised the two larger and roughly equal populations whereas a smaller part constituted adCINs (Fig. $2 D, F$ ). The three different populations were all severely affected in the Netrin-1 mutants; aCINs (green) were reduced to $17 \%$ compared with controls, dCINs (red) to $13 \%$ and adCINs (yellow) to $12 \%$, respectively (Fig. $2 \mathrm{E}, \mathrm{G}, \mathrm{H}$ ). The CINs remaining in $N t n 1^{G t / G t}$ mice detected by both tracing approaches were predominantly distributed in the ventromedial area but some more dorsal CINs were also found. Together, the majority of commissural axons do not reach the floor plate in $N t n 1^{G t / G t}$ mice, as confirmed by the severe reduction of CINs found in E12.5 embryos and also manifested at $\mathrm{P} 0$ with regard to both the number and location of the remaining CINs.

\section{Netrin mutant mice show strict synchrony in left-right coordination}

Next we went on to study the functional consequence on locomotor behavior from the found severe reduction of CINs in the spinal cord of Netrin-1 mutant mice. Due to the early lethality shortly after birth of Netrin-1 mutant mice, most likely due to an inability to suckle and feed (Serafini et al., 1996), the spinal CPG networks were analyzed in fictive locomotion preparations at P0 (Fig. 3). Isolated spinal cords were exposed to NMDA, 5HT, and 
dopamine, at concentrations that induced locomotor-like activity as revealed by suction electrode recordings of the L2 and L5 ventral roots (Fig. 3C). Wild-type and heterozygous mice displayed normal rhythm with alternation both between the left-right sides and between L2/L5 roots (Fig. $3 A$ ). In contrast, spinal cords from $N t n 1^{G t / G t}$ mice displayed synchronous rhythms between the left and right roots of the same lumbar level in all animals tested $(n=7)$, whereas the outputs between the L2 and L5 roots were normally alternating $(n=6)$ (Fig. $3 B)$. The mean period time of $2.5 \mathrm{~s}$ (mean value of 15 random cycles) of $N t n 1^{G t / G t}$ mice did not differ from littermate control mice (Student's $t$ test, $p=0.73 ; N t n 1^{G t / G t} n=7$, control $n=10)$. In both groups, we observed a tendency of prolonged bursting in the L5 roots compared with the L2 roots, as indicated by the rightward shift of data points in the L2/L5 measurements (Fig. $3 A, B$ ). Initial observations of "paddling" movements have been made in forelimbs of newborn $N t n 1^{G t / G t}$ mice (Serafini et al., 1996) while our fictive locomotion data refers to hindlimb movements; therefore, both forelimbs and hindlimbs seems similarly affected by the Netrin-1 mutation. Thus, our data show that a severe reduction of Netrin-1 guided CINs is accompanied by a pronounced synchrony in left-right locomotor-related activity.

\section{The remaining commissural} interneurons in Netrin-deficient mice are predominantly excitatory

In an effort to further characterize the aberrant locomotor behavior in $N t n 1^{G t / G t}$ mice we investigated the neurotransmitter profile of the neuronal connections over the spinal cord midline. First we examined the neurotransmitter phenotype of the CINs remaining in $N t n 1^{G t / G t}$ mice by in situ hybridization. For this purpose, we combined tracing in P0 spinal cords with in situ hybridization detecting mRNA from the dominating vesicular glutamate transport in the spinal cord (VGLUT2) or the vesicular inhibitory amino acid transporter (VIAAT) to identify excitatory and inhibitory CINs (Fig. 4). In control animals, we found $42.5 \%(n=506)$ and $60.3 \%(n=393)$ traced CINs also positive for Vglut2 or VIAAT, respectively (Fig. $4 A, C, E)$. In Ntn $1^{G t / G t}$ mice, we found 63.9\% $(n=147)$ Vglut2 and $28.2 \%(n=110)$ VIAAT-positive CINs (Fig. $4 B, D, E)$, indicating that significantly more excitatory than inhibitory CINs remain in Netrin-1 mutant mice (ratio of 0.6 versus 2.4 ).

Previous studies of simulated locomotor behavior have shown that the balance between excitation and inhibition over the midline can be influenced by the addition of the glycine reuptake inhibitor sarcosine (Kullander et al., 2003). To test the possibility that the synchronous locomotor phenotype we observed in spinal cords from $N t n 1^{G t / G t}$ mice could be restored to alternation, the glycine reuptake inhibitor sarcosine and/or the GABA reuptake
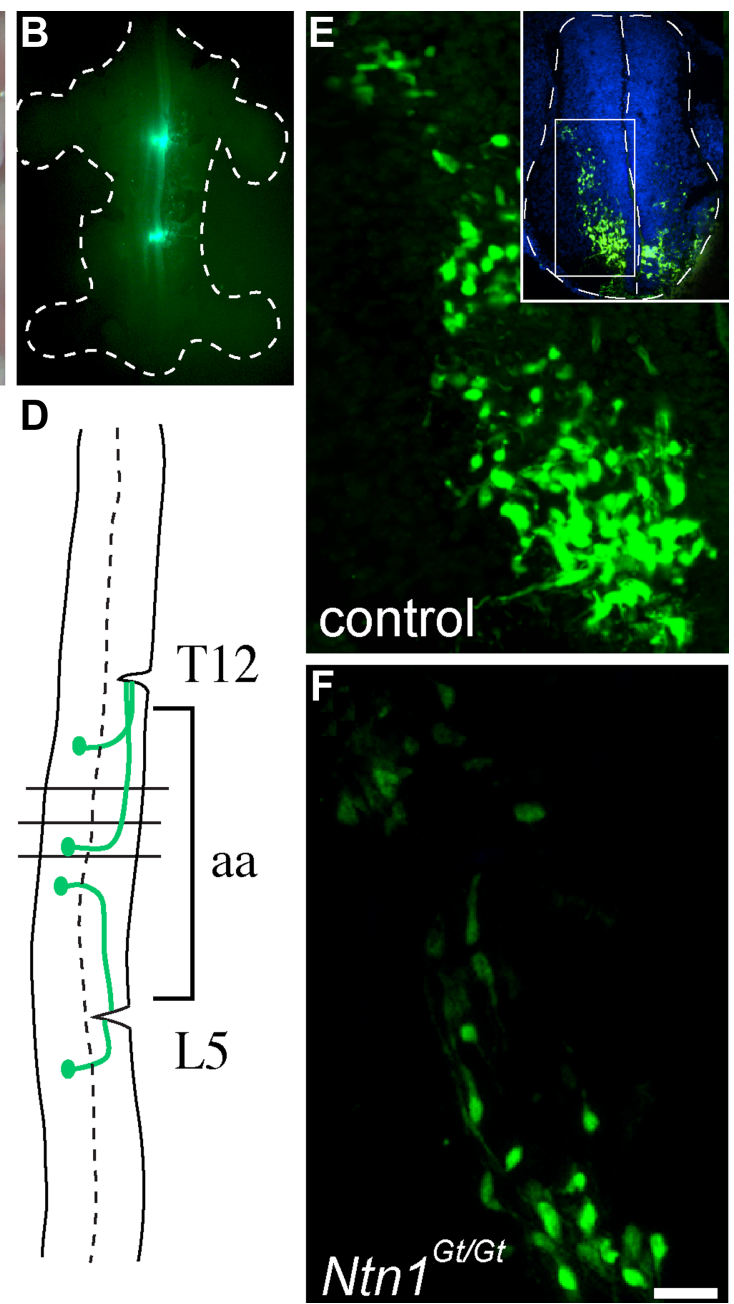

Ntn1

Figure 1. Severe loss of traced commissural interneurons in E12.5 Ntn $1^{6 t / G t}$ embryos. $A-D$, Overview of CIN of tracings in E12.5 intersegmental FDA traced CINs, from control $(\boldsymbol{F})$ and $N$ tn $1^{G t / G t}$ mice $(\boldsymbol{F})$ taken from transverse spinal cord section as indicated in the inset. White dashed line outlines the spinal cord and the white box represents area of higher-magnification picture. inhibitor nipecotic acid (NA) was added to the in vitro preparation. When analyzing the left-right coordination before and after exposure to sarcosine, no difference was detectable in the $N t n 1^{G t / G t}$ animals (Fig. $4 H$ and data not shown; sarcosine $n=4$; sarcosine + NA $n=2$; NA $n=1)$. These observations suggested that the balance between excitatory Vglut2-positive CINs and inhibitory VIAAT-positive CINs shifted toward increased excitation in Netrin-1 mutant mice and that pharmacological strengthening of inhibition was not sufficient to restore normal CPG alternation. This could possibly indicate that the remaining small number of inhibitory fibers, even if their activity was pharmacologically strengthened, was not enough to overcome excitatory contralateral input.

Axon guidance of developmental commissural interneuron subtypes is differentially affected in Netrin-1-deficient mice To further characterize the remaining CINs in Netrin-1 mutant mice, we next investigated their developmental origin using antibodies targeted against transcription factors combined with embryonic tracing (Fig. 5). Based on earlier findings, five of the 

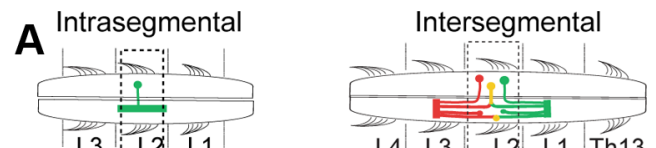
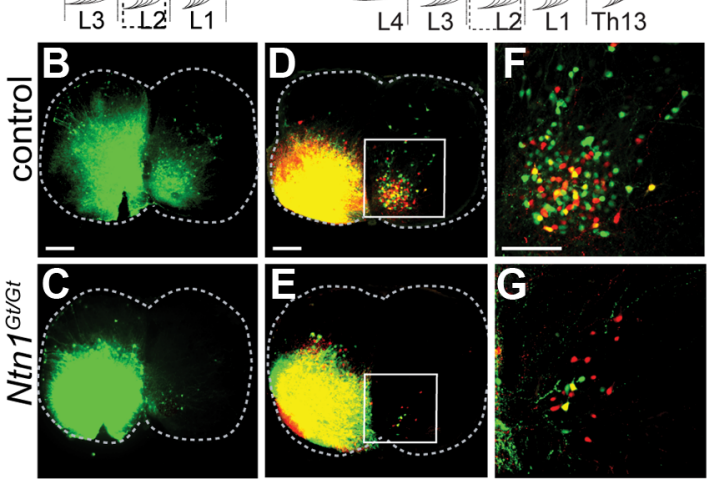

\section{H}

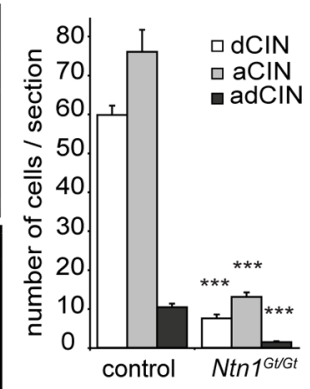

Figure 2. Loss of commissural interneurons in newborn $N t n 7^{G t / G t}$ mice. A, Overview of intrasegmental and intersegmental tracings of CINs in PO mice. $\boldsymbol{B}-\mathbf{G}$, Photomicrographs of transverse sections of the spinal cord L2 segment from control and Ntn 1 Gt/Gt mice. Low power $(\boldsymbol{B}-\boldsymbol{E})$ and higher magnification $(\boldsymbol{F}, \boldsymbol{G})$. Scale bars $200 \mu \mathrm{m}$. $\boldsymbol{H}$, Quantification of aCINs (green), dCINs (red) and adCINs (yellow). Values are given as means \pm SEM, count on 5 consecutive sections for $n=6$ animals for each group, ${ }^{* * *} p<0.0001$. The number of commissural neurons is dramatically reduced in $\mathrm{P} 0 \mathrm{Ntn} 1^{G t / G t}$ mice. White boxes indicate areas of higher magnification. Error bars indicate SEM.
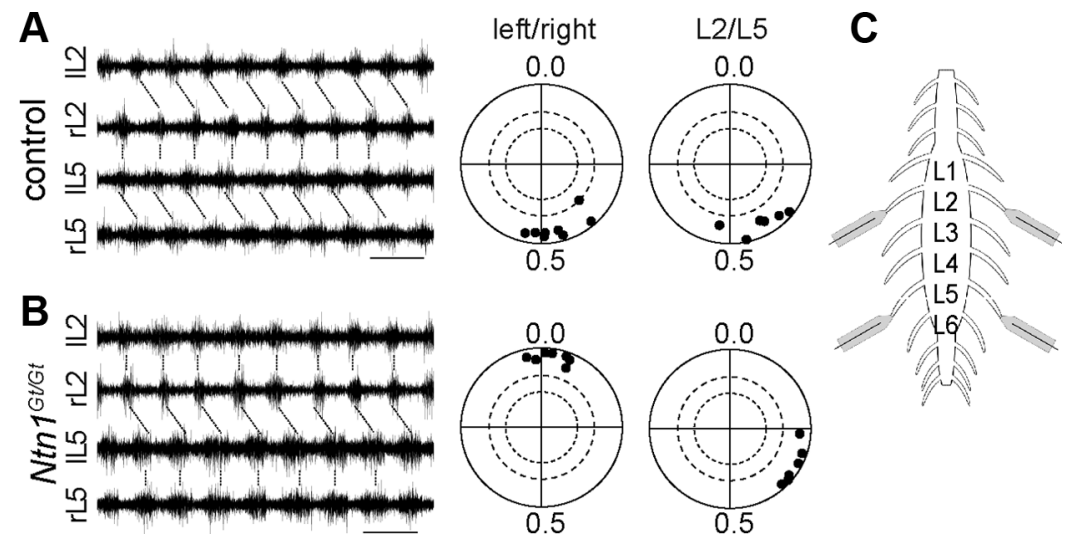

Figure 3. $N \operatorname{tn} 1^{G t / G t}$ mice display synchronous left-right coordination in fictive locomotion. $\boldsymbol{A}, \boldsymbol{B}$, Recorded activity in ventral roots of left (I) and right (r) lumbar segment (L)2 and L5 from PO spinal cords of control mice $(A)$ and Ntn ${ }^{G t / G t}$ mice (B). Control mice displayed normal left-right alternation $(n=10)$ whereas $N t n 1^{G t / G t}$ mice displayed left-right synchrony $(n=7)$. Circular phase diagrams of intersegmental L2/L5 coordination show alternation in both control $(n=8)$ and $N \operatorname{tn} 1^{G t / G t}(n=6)$ mice. Scale bar $5 \mathrm{~s}$. Each dot represent the mean phase of one animal and stippled circle indicate significant (inner) or highly significant (outer) values. C, Schematic illustration of ventral root recording set-up to measure fictive locomotion.

dorsal progenitor cell populations ( $\mathrm{dI} 1, \mathrm{dI} 2, \mathrm{dI} 3$, dI5, and dI6) and two classes of interneurons generated from the ventral progenitor cells (V0 and V3) are possible CIN candidates (Bermingham et al., 2001; Moran-Rivard et al., 2001; Pierani et al., 2001; Gross et al., 2002; Lanuza et al., 2004). Brn3a and Lbx1 were used to examine the dorsal most dI1-dI3 interneurons, as these neurons can be identified by the expression of Brn3a and absence of Lbx1(Gross et al., 2002) showing significantly fewer ${\mathrm{Brn} 3 \mathrm{a}^{+} \text {(immunopositive)/Lbx1 }}^{-}$(immunonegative) CINs in $N t n 1^{G t / G t}$ embryos (24\% compared with controls, Fig. 5C,D,L). CINs choose different trajectories after crossing the midline (Imondi and Kaprielian, 2001; Kadison and Kaprielian, 2004), which could influence the efficiency of our tracing technique. For example, some CINs originating from the $\mathrm{dI} 1$ and $\mathrm{dI} 2$ domains have been shown to diverge directly to the lateral funiculus while others project several segments alongside the floor plate (Reeber et al.,2008; Avraham et al., 2009). However, using our approach we detected at least part of these dorsal subpopulations (supplemental Fig. S1, available at www.jneurosci.org as supplemental material). These results indicate that dorsally originating $\mathrm{dI} 1-\mathrm{dI} 3$ neurons are highly dependent on Netrin-1-mediated axon guidance.

Simultaneous detection with Lbx1 and Pax2 immunohistochemistry allowed us to investigate dI4-dI6 cells (Gross et al., 2002; Müller et al., 2002; Cheng et al., 2004) (Fig. 5E,F). DI5 neurons are identified by the expression of Lbx1 and absence of Pax2 (Gross et al., 2002). In contrast, both dI4 and dI6 are identified by the coincident expression of Lbx1 and Pax2, but since earlier work have indicated that dI4 cells develop into association interneurons of the substantia gelatinosa rather than CINs (Gross et al., 2002), we assume that the CINs identified by Lbx1 and Pax2 double labeling arise from dI6 progenitors. Lbx $1^{+} / \mathrm{Pax} 2^{+/-}$cells were found in the ventromedial and more dorsolateral populations of the traced CINs (Fig. $5 E, F)$. The absolute numbers of the Lbx $1^{+} / \mathrm{Pax} 2^{+/-}$traced populations were significantly reduced to $25 \%$ and $26 \%$, respectively in $N t n 1^{G t / G t}$ embryos compared with controls (Fig. $5 L$ ). The axonal projection pattern of dI5 interneurons have not previously been reported, although earlier studies have shown that these neurons settle in a cluster dorsal to the motor column in the intermediate spinal cord (Gross et al., 2002), in an area where the most dorsal ascending CINs reside. DI6 interneurons migrate to the ventral spinal cord and settle in lamina VIII, an area in which the majority of local CINs can be found (Gross et al., 2002; Nissen et al., 2005). Indeed, our data suggest that both $\mathrm{Lbx}_{1}{ }^{+} / \mathrm{Pax} 2^{-}$and $\mathrm{Lbx}^{+} / \mathrm{Pax} 2^{+}$ cells are at least partly commissural, settle in ventral regions, and are highly dependent on Netrin-1mediated axon guidance (Fig. $5 L$ ).

With Pax2, Evx1, and Lbx1 immunohistochemistry, the V0 ventral subpopulations were investigated (Briscoe et al., 1999; Moran-Rivard et al., 2001). Dbx1 ${ }^{+}$progenitor cells give rise to two $\mathrm{V} 0$ subpopulations, $\mathrm{V} 0_{\mathrm{v}}$ and $\mathrm{V} 0_{\mathrm{d}}$, where $\mathrm{V}_{\mathrm{v}}$ can be identified by the expression of Evx1. Additionally, it has been suggested that V0, express Pax2 (Burrill et al., 1997). However, we have observed that they express Pax2 protein at very low levels as they are generated and migrate ventrally (Fig. 6); thus, most likely not being detected as $\mathrm{Pax} 2^{+}$neurons in our tracing experiments. In contrast, $\mathrm{V} 0_{\mathrm{d}}$ neurons, generated just dorsal to $\mathrm{V} 0_{\mathrm{v}}$ neurons and ventral to Lbx1-expressing dI6 neurons, appear to express Pax2 at high levels (Fig. 6). As these neurons lack a specific marker, we used Pax2 in combination with Lbx1 to distinguish ventral Lbx1 ${ }^{-} \mathrm{V} 0{ }_{\mathrm{d}}$ neurons from the dorsal Lbx $1^{+} / \mathrm{Pax} 2^{+}$dI6 neurons. In the $\mathrm{Pax} 2^{+} / \mathrm{Lbx}^{-}$population, we detected a significant de- 

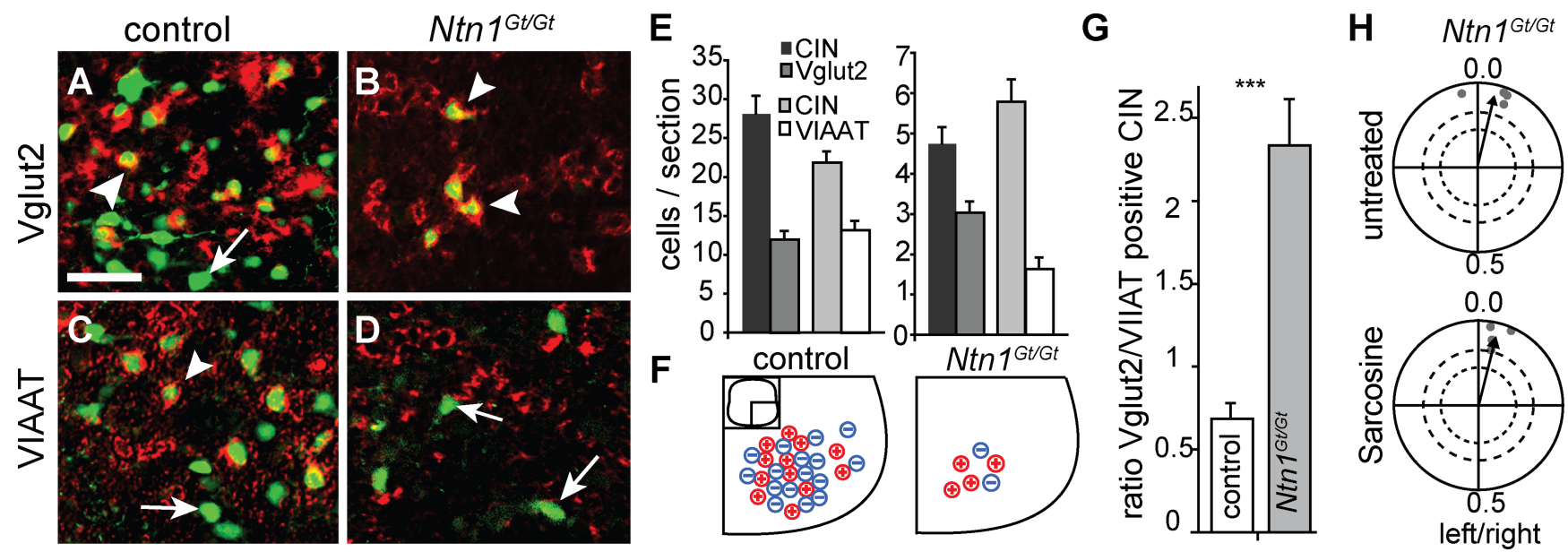

Figure 4. Remaining commissural interneurons in Ntn $7^{G / G t}$ mice are predominantly excitatory. A-D, Photomicrographs of in situ staining for Vglut2 or VIAAT mRNA on FDA traced spinal cord sections. Arrowheads show CINs positive for Vglut2 or VIAAT respectively, while arrows indicate single labeled CINs. Scale bar $25 \mu \mathrm{m}$. E, Quantification of the neurotransmitter used by traced CINs on lumbar spinal cord sections from control or Ntn ${ }^{G t / G t}$ mice, counted on $6-10$ consecutive sections and $n=3$ animals for each group. $\boldsymbol{F}$, Schematic drawing illustrating glutamatergic (red) or glycinergic/GABAergic (blue) neurotransmitter phenotype of commissural neurons in control and Ntn $7^{G / G t}$ mice. G, The ratio of excitatory versus inhibitory CINs for control, Ntn $7^{G t / G t}$ mice from data presented in $(\boldsymbol{E})$ showing a shift toward more Vglut2-positive CINs in Ntn $1^{G / / G t}$ mice. Values are given as means \pm SEM, ${ }^{* * *} p<0.001$. H, Circular phase diagrams of analyzed fictive locomotion experiments performed on spinal cords from Ntn $1^{G t / G t}$ mice before and after treatment with $100 \mu \mathrm{m}$ sarcosine. The arrows represent the grand mean of all animals in each condition. Only animals that subsequently were treated are included in the diagrams $(n=4)$. Sarcosine treatments left the synchronous left-right coordination unchanged in the Ntn ${ }^{G t / G t}$ mice.

crease in the average number of traced neurons per section, similar to that in more dorsal originating CIN, to $20 \%$ remaining CINs in $N t n 1^{G t / G t}$ embryos compared with controls (Fig. $5 \mathrm{~L}$ ). However, for the $\mathrm{Evxl}^{+}$population we found $45 \%$ of labeled CINs remaining in $N t n 1^{G t / G t}$ embryos compared with controls. Thus, our data show that V0 neurons are at least partly commissural, as also suggested earlier (Moran-Rivard et al., 2001; Pierani et al., 2001; Lanuza et al., 2004), and that the Evx1 ${ }^{+}$population is less severely affected than the $\mathrm{Pax} 2^{+} / \mathrm{Lbx} 1^{-}$population in $N t n 1^{G t / G t}$ embryos.

Finally, we investigated the ventralmost V3 interneurons, which derive from Nkx2.2-expressing progenitor cells and later on also express Sim 1 (Briscoe et al., 1999). Interestingly, the same numbers of $\mathrm{Nkx} 2.2^{+}$neurons were found among the traced CINs in both control and $N t n 1^{G t / G t}$ embryos (Fig. $5 I, J, L$ ). Our data demonstrate that ventral V3 interneurons project axons over the midline and, strikingly, that the Nkx2.2 ${ }^{+}$commissural V3 interneurons seem completely unaffected by the loss of Netrin at the spinal cord midline.

Together, our data show that there is a gradient of CIN numbers originating from the ventral to dorsal progenitor domains, where the most ventral V3 population seems to be completely independent on Netrin-1 while the dorsal-originating neurons are highly dependent on Netrin-1 signaling to properly find and cross the midline.

\section{Discussion}

Synchronous locomotion in Netrin-1 mutant mice

Here we show that neuronal circuit formation underlying normal left-right alternation during fictive locomotion is dependent on Netrin-1-mediated axon guidance in the spinal cord. Netrin-1 represents the first example of an axon guidance molecule that when dysfunctional, completely switches the alternating leftright coordination to strict synchrony in the neonatal spinal cord. Earlier studies of mice deficient of EphA4 and ephrinB3 have shown a synchronous gait in adult animals, whereas their phenotype in the fictive locomotion setup vary between alternation and synchrony (Kullander et al., 2003). In addition, studies of RacGAP $\alpha 2$-chimaerin, an EphA4 downstream signaling molecule, have reported either uncoordinated or synchronous activities in neonatal mice (Beg et al., 2007; Wegmeyer et al., 2007). In conclusion, aberrant midline crossing of excitatory ipsilateral spinal cord interneurons in EphA4 mutant mice disrupts the normal balance between excitation and inhibition over the midline (Kullander et al., 2003) while in $N t n 1^{G t / G t}$ mice, a severe reduction of CINs, representing an opposite scenario to ephA4 $4^{-1-}$ mice, eventually results in a similar neurotransmitter imbalance.

Both CINs and ipsilateral neurons play important roles during locomotion and, likewise, axons from both populations can be guided by Netrin-1 (Saueressig et al., 1999). Thus, the synchronous locomotor output observed in $N t n 1^{G t / G t}$ mice could in principle be due to misprojections of ipsilateral axons or abnormally projecting CIN on the ipsilateral side. V1 ipsilateral interneurons, which form direct connections to motor neurons, have been shown to regulate the speed of locomotion in neonatal fictive locomotion (Gosgnach et al., 2006). Deletion and silencing of V2a interneurons, which make ipsilateral excitatory Vglut2 contacts to multiple CIN populations, results in greater variability in cycle period and amplitude of locomotor bursts (Crone et al., 2008). However, these ipsilateral population associated locomotor phenotypes were not observed in Netrin-1 mutant mice and, in addition, ipsilateral coordination between lumbar level L2 and L5 is normal, suggesting that the strict left-right synchronous locomotor activity is more likely to be related to the role Netrin-1 plays in guiding CINs to the midline.

\section{Netrin-1 preferentially guides dorsally originating interneurons}

At E12.5, a severe reduction of spinal CINs was detected in mice lacking Netrin-1, which was expected considering the reduced amount of fibers crossing the midline in this mouse mutant (Serafini et al., 1996). Netrin-1 is a potential attractant for fibers from cells originating from both the dorsal and ventral neuroepithelium to the floor plate. Our data, together with previously published results, suggest that CINs can be found within the dI1dI3, dI5, dI6, V0, and V3 subpopulations of neurons. Of these, $\mathrm{dI} 1-\mathrm{dI} 3, \mathrm{dI} 5, \mathrm{dI} 6$ and $\mathrm{V} 0_{\mathrm{d}}$ cells were severely affected in Netrin-1 mutant mice, indicating a strong dependence on Netrin-1 to 

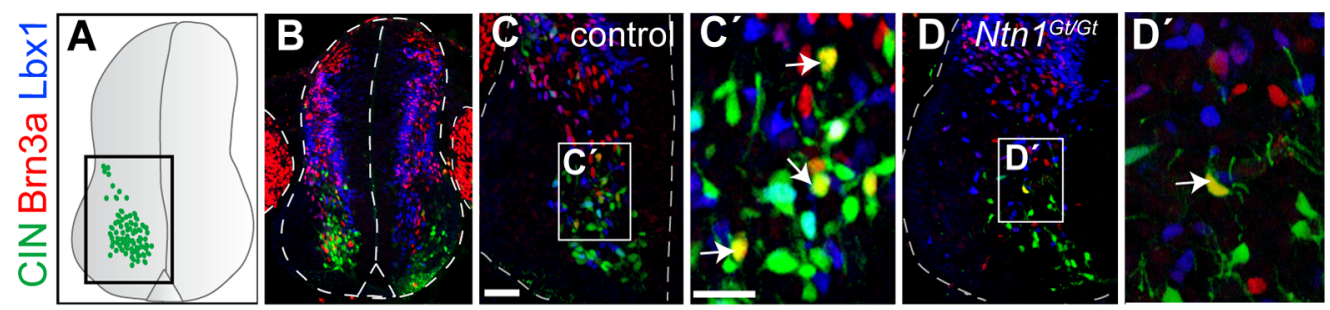

K
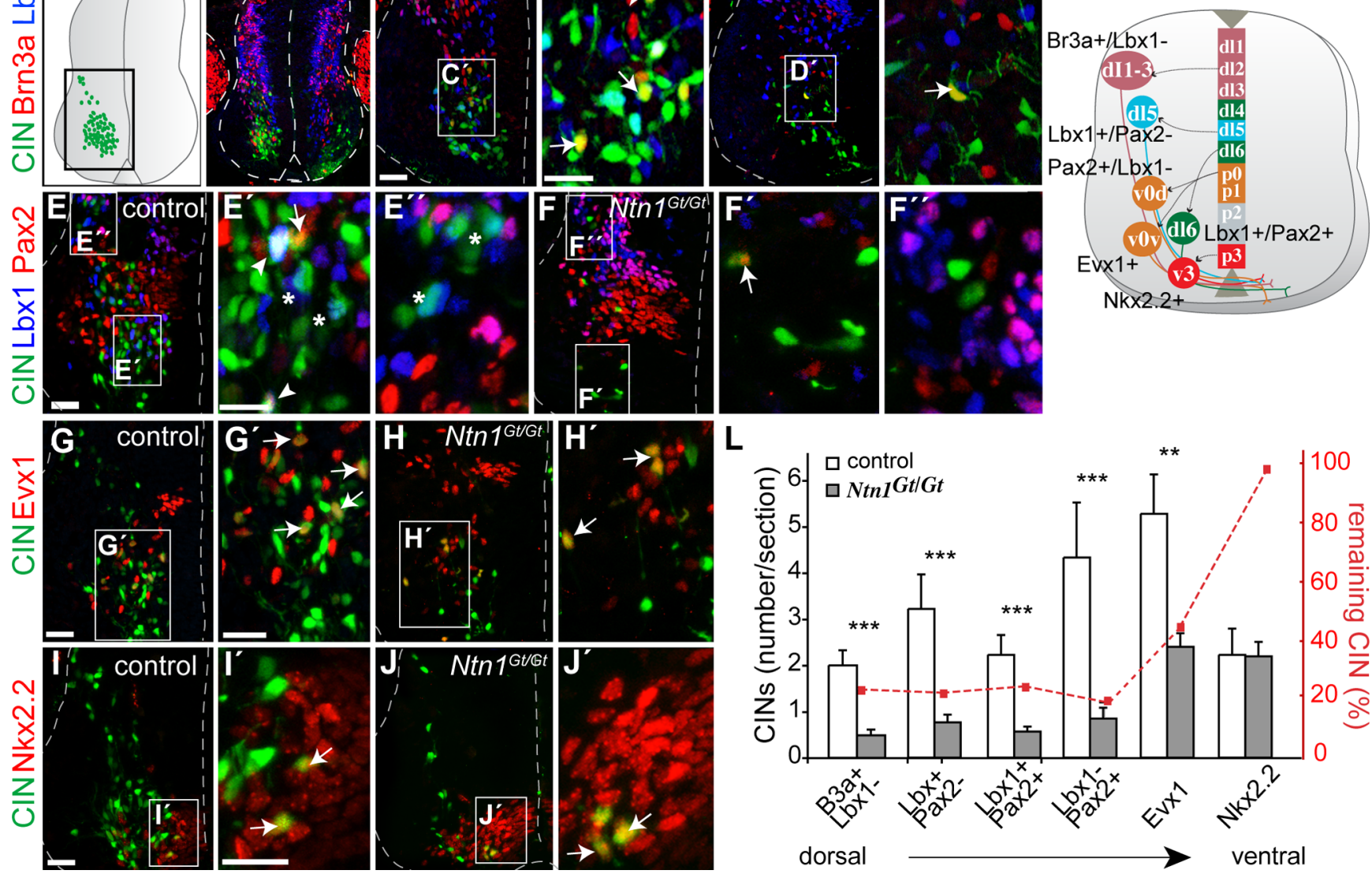

Figure 5. Loss of Netrin-1 has a more severe effect on dorsal compared with ventral originating CIN subpopulations. A, B, Schematic representation of the E12.5 neuronal tube and the position of traced CINs according to the transverse immunostained section of an FDA-traced control embryo shown in B. C-J, Photomicrographs of control and Ntn $1^{G / / G t}$ animals showing the region of traced CINs according to the black box in $\boldsymbol{A}$ and staining for transcription factors as indicated to the left. White boxes in $\boldsymbol{C}-\boldsymbol{J}$ indicate areas of higher magnification in respective panel to the right $\left(\boldsymbol{C}^{\prime}-\boldsymbol{J}^{\prime}\right)$.

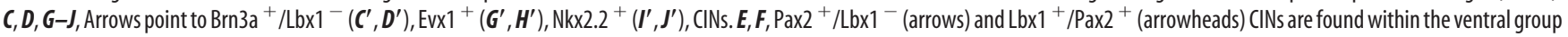
of traced neurons $\left(\boldsymbol{E}^{\prime}\right)$ while the majority of $\mathrm{Lbx} 1^{+} / \mathrm{Pax} 2^{-}$CINs (stars) are more dorsally located $\left(\boldsymbol{E}^{\prime \prime}\right)$. Scale bars, $30 \mu \mathrm{m} . \boldsymbol{K}$, Schematic drawing of progenitor domains and early neuronal domains labeled by the transcription factors used in this study. L, Quantification of transcription factor phenotype in traced CINs shows significant differences in affected CIN populations in $N$ tn $7^{G / / G t}$ mice. CIN remaining in $N t n 1^{G t / G t}$ animals colabel predominantly with Evx1 and Nkx2.2. Error bars indicate SEM $n=5$ for control and $n=6$ for Ntn $1^{G t / G t}$ embryos for all markers analyzed. For Brn3a/Lbx1 measurements in control embryos; $T$ (total count traced neurons) $=724, s$ (sections) $=19 ;$ for Ntn $1^{G t / G t}$ embryos: $T=460, s=39 ; * * * 00.0001$. Lbx $1 /$ Pax 2 measurements in control embryos; $\mathrm{T}=555, \mathrm{~s}=15 ;$ for Ntn $1^{G t / G t}$ embryos: $\mathrm{T}=533, \mathrm{~s}=46 ; p<0.0001$. Evx1 measurements in control embryos: $\mathrm{T}=541, \mathrm{~s}=18 ; \mathrm{Ntn} 1^{G t / G t}$ embryos: $\mathrm{T}=569, \mathrm{~s}=50 ;{ }^{* *} p=0.0033 . \mathrm{Nkx2.2}$ in control embryos: $\mathrm{T}=332, \mathrm{~s}=11 ; \mathrm{Ntn} 1^{\mathrm{Gt} / \mathrm{Gt}}$ embryos: $\mathrm{T}=586, \mathrm{~s}=51 ; p=0.9731$.

properly find the midline. We observed a minor loss of $\mathrm{VO}_{\mathrm{v}}$ and strikingly, the most ventral V3 CINs are completely unaffected by the lack of Netrin-1, suggesting a different mechanism to approach the midline, for example via other guidance molecules, such as Sonic Hedgehog (Charron et al., 2003). Alternatively, populations sufficiently close to the ventral floor plate may randomly project an adequate number of fibers during development to establish normal circuit formation. In any case, our observations suggest a ventral to dorsal increase of Netrin-1 dependence for CINs.

\section{A shift toward excitation over the midline in Netrin-1 mutant mice}

The neurotransmitters glycine and GABA provide the necessary fast synaptic crossed inhibition for deactivation of the contralateral side during locomotion (Cowley and Schmidt, 1995). However, it has also been reported that CINs consist of both excitatory and inhibitory populations (Cohen and Harris-Warrick, 1984; Grillner, 2003). Recent studies in rat and mice spinal cord have shown, that excitatory CIN terminals are less abundant than inhibitory ones (Wéber et al., 2007; Restrepo et al., 2009). This is comparable to our quantification of excitatory (Vglut ${ }^{+}$) and inhibitory $\left(\mathrm{VIAAT}^{+}\right.$) CIN cell bodies in spinal cord of wild-type mice (Fig. 5). In Netrin-1 mutant mice, more excitatory than inhibitory CINs remained, resulting in the converse situation with a shift toward more excitatory signals being transmitted over the midline. CINs are thought to make up two parallel systems that control left-right coordination. (1) A left-right alternation circuit, that consists of inhibitory CINs, which monosynaptically inhibit contralateral motor neurons (MNs), and a set of excitatory CINs that indirectly inhibit MNs by activating contralateral inhibitory interneurons. This system is believed to be necessary for contralateral silencing during left-right alternating locomotion. (2) A left-right synchrony circuit, consisting of a set of excitatory CINs directly activating contralateral MNs (Nishimaru et al., 2006; Quinlan and Kiehn, 2007). The latter has been proposed to be active e.g., during hopping probably by overriding the alternation circuitry. In addition, application of the glycine receptor inhibitor strychnine to mouse spinal cords with uncoordinated (E15) or alternating (E18 and older) activity, has been shown to transform the activity to synchrony (Branchereau et al., 2000). This suggests that inhibitory signaling is important for 
normal left/right alternation, and identifies the presence of a bilateral synchronous bursting activity independent of glycinergic signaling, which, under normal conditions, is suppressed. The synchronous coordination observed in Netrin-1 mutant mice suggest that the excitatory CIN activity remaining in these mice is more important for general cross-midline activation or left-right synchrony than for maintenance of left-right alternation. We therefore propose that the strictly synchronous activity in Netrin-1 mutant mice is due to the loss of the major parts of the alternation circuitry whereas the synchrony circuitry is largely left intact (Fig. 7).

\section{A role for ventral derived commissural interneurons in left-right synchrony}

Disruption of Netrin-1-mediated axon guidance leads to a relative gain of excitatory CINs, explained at least in part by the unaffected population of excitatory $\mathrm{V} 3$ originating neurons. Mice in which the V3 derived neurons were genetically manipulated to disrupt their synaptic transmission maintained the ability of leftright alternation (Zhang et al., 2008). In contrast, in Netrin-1 mutant mice, the only CIN population left intact in its entirety was the V3 interneurons. Despite the severe overall loss of CINs, the remaining neurons appear sufficient to maintain a coordinated activity over the midline, albeit with a strict left-right synchronous pattern. This suggests that the V3 population is an important component of the left-right synchrony circuitry, which is in line with previous findings where V3 neurons were suggested to balance the motor outputs produced in each half of the spinal cord (Zhang et al., 2008).

Neurons originating from the V0 population, which was only partially affected, could in principle also be involved in left-right synchrony. The V0 population consist of $70 \%$ inhibitory and $30 \%$ excitatory interneurons (Lanuza et al., 2004). Our data suggest that Evx1 ${ }^{+} \mathrm{V} 0_{\mathrm{v}}$ neurons express lower or no levels of Pax2 protein while $\mathrm{V} 0_{\mathrm{d}}$ neurons express high levels of Pax2 (Fig. 6). Pax2 has been shown to mark an inhibitory cell fate in dorsal neurons (Cheng et al., 2004), which if this applies also to the V0 population, would suggest that V0d neurons are inhibitory, while $\mathrm{VO}_{\mathrm{v}}$ neurons would constitute the majority of the excitatory V0 neurons. This is consistent with the observed shift toward excitation in Netrin-1-deficient mice, since more $\mathrm{V}_{\mathrm{d}}$ neurons lose contralateral contacts compared with $\mathrm{V}_{\mathrm{v}}$ neurons ( $80 \%$ vs $55 \%$ ). Interestingly, the $\mathrm{V}_{\mathrm{d}}$ population has earlier been associated to mouse spinal cord CPG function (Lanuza et al., 2004). Mice that lacked Dbx1 lost both the $\mathrm{V} 0_{\mathrm{v}}$ and $\mathrm{V} 0_{\mathrm{d}}$ populations, resulting in intermittent episodes of synchrony between left and right ventral roots. However, in mice lacking Evx 1 , only $\mathrm{V} 0_{\mathrm{v}}$ interneurons were lost, with no effect on the normally alternating fictive locomotion pattern. Since the phenotype of Netrin-1 mutant mice is different from Dbx1 mutants in that it is consistently left-right synchronous, we postulate that the $\mathrm{V} 0_{\mathrm{v}}$ population, in addition to the V3 population, could be part of the left-right synchrony circuit while $\mathrm{V} 0_{\mathrm{d}}$ and additional dorsally originating CIN subpopulations could be vital for normal left-right alternation (Fig. 7).

With regard to dorsally originating CINs, dI1 interneurons are suggested to compose the spinocerebellar tract (Bermingham
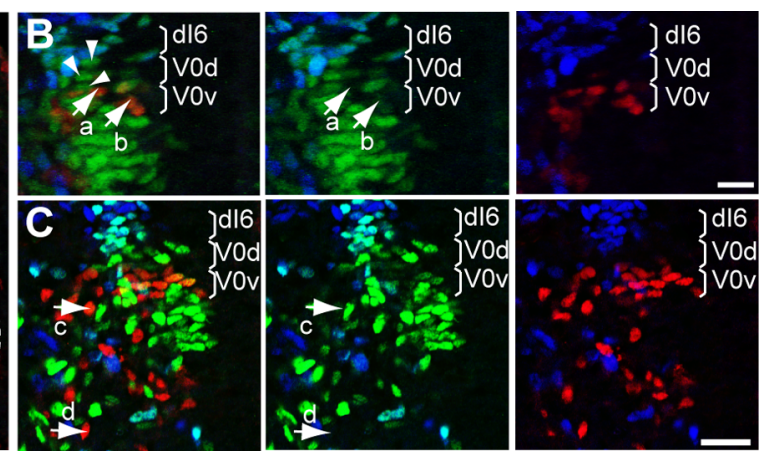

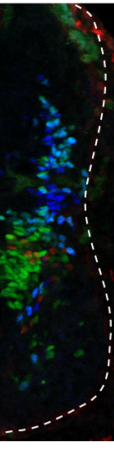

Figure 6. $\quad V_{\mathrm{d}}$ neurons express Pax2. $A-C$, Photomicrographs of immunohistochemistry experiments using antibodies against indicated transcription factors on E11.5 $(\boldsymbol{A}, \boldsymbol{B})$ or E12.5 $(\boldsymbol{C})$ spinal cord transverse sections. White box in $\boldsymbol{A}$ indicates areas of higher magnification in $\boldsymbol{B}$ panels. Arrowheads indicate Pax2 ${ }^{+}$cells and arrows indicate Evx1 ${ }^{+}\left(\mathrm{V}_{\mathrm{v}}\right)$ cells. AtE11.5, cells in the ventral V0 V0 subpopulations. $\boldsymbol{C}, 0$ ne day later, a similar situation is observed; $V_{v}$ neurons, as labeled by Evx 1 (arrows $\mathbf{c}$ and $\boldsymbol{d}$ ), express lower or no levels of Pax2 protein while V0 ${ }_{d}$ neurons express high levels of Pax2. Scale bars: $A, 100 \mu \mathrm{m}, \boldsymbol{B}, \boldsymbol{C}, 30 \mu \mathrm{m}$.

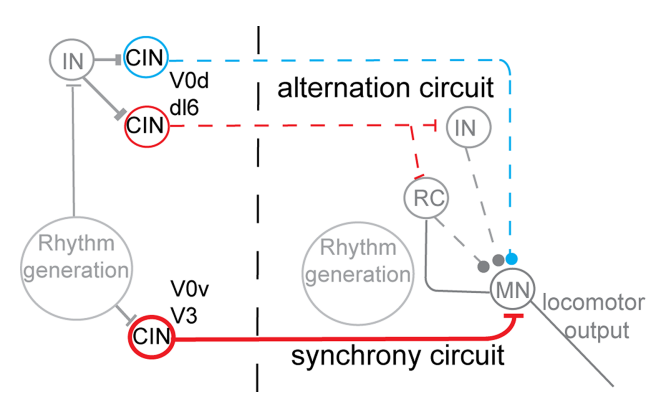

Figure 7. Schematic model of the $C P G$ components responsible for left-right coordination in the Netrin-1 mutant spinal cord (adapted from Crone et al., 2008). Due to the loss of commissural interneurons from distinct progenitor domains in netrin-1 mutant mice, the left-right alternation circuit is weakened as indicated by stippled lines, compared with the left-right synchrony circuit (solid line). This reduction of CINs results in a loss of more inhibitory (blue) than excitatory (red) fibers over the midline, which is a likely explanation for the left-right synchrony observed in fictive locomotion of Netrin-1 mutant mice. The model also includes possible subpopulation identities of ventral and dorsal derived commissural interneurons within the spinal locomotor network, based on the present findings and prior work (Lanuza et al., 2004; Zhang et al., 2008).

et al., 2001). DI2 interneurons seem to be a mixed population consisting of ipsilateral interneurons and CINs that accumulate where the neurons of the spinothalamic tract reside (Gross et al., 2002) and are therefore not likely to contribute to CPG activity. For dI3 neurons the situation is less clear, although unpublished observations indicate that these neurons may contribute to ascending pathways (Gross et al., 2002). DI5 neurons settle in the most dorsolateral part of lamina VII (this study) and likely constitutes an excitatory population of interneurons since they have been found to be Pax2- and Tlx1/3+ (Cheng et al., 2004). Based on their final location in the ventral spinal cord, dI6 neurons are potentially involved in CPG coordination and because of the severe shift toward less inhibitory CINs in Netrin-1 mutant mice, we speculate that the commissural inhibitory dI6 neurons are vital components for production of alternating left-right CPG activity.

Previous studies focused on interneurons originating in the ventral spinal cord (Lanuza et al., 2004; Crone et al., 2008; Zhang et al., 2008), and our results stress the importance of extending future studies to investigate neurons derived also from the dorsal neuroepithelium. Additionally, studies of cells expressing Netrin receptors and their potential participation in locomotor CPG 
circuits will be also be relevant to resolve the characteristics of the neurons coordinating left-right alternation and left-right synchrony in mammals and, in more general terms, how interneurons behave to master and coordinate neuronal circuitries.

\section{References}

Avraham O, Hadas Y, Vald L, Zisman S, Schejter A, Visel A, Klar A (2009) Transcriptional control of axonal guidance and sorting in dorsal interneurons by the Lim-HD proteins Lhx9 and Lhx1. Neural Dev 4:21.

Beg AA, Sommer JE, Martin JH, Scheiffele P (2007) alpha2-Chimaerin is an essential EphA4 effector in the assembly of neuronal locomotor circuits. Neuron 55:768-778.

Bermingham NA, Hassan BA, Wang VY, Fernandez M, Banfi S, Bellen HJ, Fritzsch B, Zoghbi HY (2001) Proprioceptor pathway development is dependent on Math1. Neuron 30:411-422.

Branchereau P, Morin D, Bonnot A, Ballion B, Chapron J, Viala D (2000) Development of lumbar rhythmic networks: from embryonic to neonate locomotor-like patterns in the mouse. Brain Res Bull 53:711-718.

Briscoe J, Sussel L, Serup P, Hartigan-O'Connor D, Jessell TM, Rubenstein JL, Ericson J (1999) Homeobox gene Nkx2.2 and specification of neuronal identity by graded Sonic hedgehog signalling. Nature 398:622-627.

Burrill JD, Moran L, Goulding MD, Saueressig H (1997) PAX2 is expressed in multiple spinal cord interneurons, including a population of EN1+ interneurons that require PAX6 for their development. Development 124:4493-4503.

Charron F, Stein E, Jeong J, McMahon AP, Tessier-Lavigne M (2003) The morphogen sonic hedgehog is an axonal chemoattractant that collaborates with netrin-1 in midline axon guidance. Cell 113:11-23.

Cheng L, Arata A, Mizuguchi R, Qian Y, Karunaratne A, Gray PA, Arata S, Shirasawa S, Bouchard M, Luo P, Chen CL, Busslinger M, Goulding M, Onimaru H, Ma Q (2004) Tlx3 and Tlx1 are post-mitotic selector genes determining glutamatergic over GABAergic cell fates. Nat Neurosci 7:510-517.

Cohen AH, Harris-Warrick RM (1984) Strychnine eliminates alternating motor output during fictive locomotion in the lamprey. Brain Res 293:164-167.

Cowley KC, Schmidt BJ (1995) Effects of inhibitory amino acid antagonists on reciprocal inhibitory interactions during rhythmic motor activity in the in vitro neonatal rat spinal cord. J Neurophysiol 74:1109-1117.

Crone SA, Quinlan KA, Zagoraiou L, Droho S, Restrepo CE, Lundfald L, Endo T, Setlak J, Jessell TM, Kiehn O, Sharma K (2008) Genetic ablation of V2a ipsilateral interneurons disrupts left-right locomotor coordination in mammalian spinal cord. Neuron 60:70-83.

Eide AL, Glover JC (1995) Development of the longitudinal projection patterns of lumbar primary sensory afferents in the chicken embryo. J Comp Neurol 353:247-259.

Gosgnach S, Lanuza GM, Butt SJ, Saueressig H, Zhang Y, Velasquez T, Riethmacher D, Callaway EM, Kiehn O, Goulding M (2006) V1 spinal neurons regulate the speed of vertebrate locomotor outputs. Nature 440:215-219.

Goulding M, Lanuza G, Sapir T, Narayan S (2002) The formation of sensorimotor circuits. Curr Opin Neurobiol 12:508-515.

Grillner S (2003) The motor infrastructure: from ion channels to neuronal networks. Nat Rev Neurosci 4:573-586.

Gross MK, Dottori M, Goulding M (2002) Lbxl specifies somatosensory association interneurons in the dorsal spinal cord. Neuron 34:535-549.

Imondi R, Kaprielian Z (2001) Commissural axon pathfinding on the contralateral side of the floor plate: a role for B-class ephrins in specifying the dorsoventral position of longitudinally projecting commissural axons. Development 128:4859-4871.

Jessell TM (2000) Neuronal specification in the spinal cord: inductive signals and transcriptional codes. Nat Rev Genet 1:20-29.

Kadison SR, Kaprielian Z (2004) Diversity of contralateral commissural projections in the embryonic rodent spinal cord. J Comp Neurol 472:411-422.
Kennedy TE, Serafini T, de la Torre JR, Tessier-Lavigne M (1994) Netrins are diffusible chemotropic factors for commissural axons in the embryonic spinal cord. Cell 78:425-435.

Kjaerulff O, Kiehn O (1996) Distribution of networks generating and coordinating locomotor activity in the neonatal rat spinal cord in vitro: a lesion study. J Neurosci 16:5777-5794.

Kullander K, Butt SJ, Lebret JM, Lundfald L, Restrepo CE, Rydström A, Klein R, Kiehn O (2003) Role of EphA4 and EphrinB3 in local neuronal circuits that control walking. Science 299:1889-1892.

Lanuza GM, Gosgnach S, Pierani A, Jessell TM, Goulding M (2004) Genetic identification of spinal interneurons that coordinate left-right locomotor activity necessary for walking movements. Neuron 42:375-386.

Moran-Rivard L, Kagawa T, Saueressig H, Gross MK, Burrill J, Goulding M (2001) Evxl is a postmitotic determinant of v0 interneuron identity in the spinal cord. Neuron 29:385-399.

Müller T, Brohmann H, Pierani A, Heppenstall PA, Lewin GR, Jessell TM, Birchmeier C (2002) The homeodomain factor lbxl distinguishes two major programs of neuronal differentiation in the dorsal spinal cord. Neuron 34:551-562.

Nishimaru H, Restrepo CE, Kiehn O (2006) Activity of Renshaw cells during locomotor-like rhythmic activity in the isolated spinal cord of neonatal mice. J Neurosci 26:5320-5328.

Nissen UV, Mochida H, Glover JC (2005) Development of projectionspecific interneurons and projection neurons in the embryonic mouse and rat spinal cord. J Comp Neurol 483:30-47.

Pierani A, Moran-Rivard L, Sunshine MJ, Littman DR, Goulding M, Jessell TM (2001) Control of interneuron fate in the developing spinal cord by the progenitor homeodomain protein Dbx1. Neuron 29:367-384.

Quinlan KA, Kiehn O (2007) Segmental, synaptic actions of commissural interneurons in the mouse spinal cord. J Neurosci 27:6521-6530.

Reeber SL, Sakai N, Nakada Y, Dumas J, Dobrenis K, Johnson JE, Kaprielian Z (2008) Manipulating Robo expression in vivo perturbs commissural axon pathfinding in the chick spinal cord. J Neurosci 28:8698-8708.

Restrepo CE, Lundfald L, Szabó G, Erdélyi F, Zeilhofer HU, Glover JC, Kiehn O (2009) Transmitter-phenotypes of commissural interneurons in the lumbar spinal cord of newborn mice. J Comp Neurol 517:177-192.

Saueressig H, Burrill J, Goulding M (1999) Engrailed-1 and netrin-1 regulate axon pathfinding by association interneurons that project to motor neurons. Development 126:4201-4212.

Schaeren-Wiemers N, Gerfin-Moser A (1993) A single protocol to detect transcripts of various types and expression levels in neural tissue and cultured cells: in situ hybridization using digoxigenin-labelled cRNA probes. Histochemistry 100:431-440.

Serafini T, Colamarino SA, Leonardo ED, Wang H, Beddington R, Skarnes WC, Tessier-Lavigne M (1996) Netrin-1 is required for commissural axon guidance in the developing vertebrate nervous system. Cell 87:1001-1014.

Stokke MF, Nissen UV, Glover JC, Kiehn O (2002) Projection patterns of commissural interneurons in the lumbar spinal cord of the neonatal rat. J Comp Neurol 446:349-359.

Wallén-Mackenzie A, Gezelius H, Thoby-Brisson M, Nygård A, Enjin A, Fujiyama F, Fortin G, Kullander K (2006) Vesicular glutamate transporter 2 is required for central respiratory rhythm generation but not for locomotor central pattern generation. J Neurosci 26:12294-12307.

Wéber I, Veress G, Szucs P, Antal M, Birinyi A (2007) Neurotransmitter systems of commissural interneurons in the lumbar spinal cord of neonatal rats. Brain Res 1178:65-72.

Wegmeyer H, Egea J, Rabe N, Gezelius H, Filosa A, Enjin A, Varoqueaux F, Deininger K, Schnütgen F, Brose N, Klein R, Kullander K, Betz A (2007) EphA4-dependent axon guidance is mediated by the RacGAP alpha2chimaerin. Neuron 55:756-767.

Zhang Y, Narayan S, Geiman E, Lanuza GM, Velasquez T, Shanks B, Akay T, Dyck J, Pearson K, Gosgnach S, Fan CM, Goulding M (2008) V3 spinal neurons establish a robust and balanced locomotor rhythm during walking. Neuron 60:84-96. 